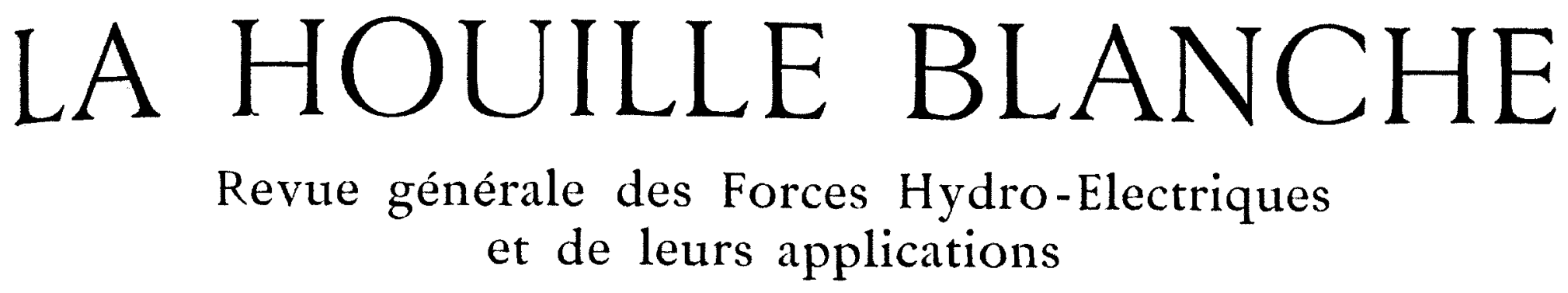

3e Année. - Mai 1904. - No 5.

\begin{abstract}
La Houille notre a fail l'Industrie moderne; la Houille blanche la transformera.
\end{abstract}

\section{Les Forces hydrauliques des Pyrénées}

Nous empruntons au magnifique volume du "COMPTE RENDT DLS TRIVAIX DU $2^{\circ}$ CONGRES DU SUD-OUEST-NAVIGABLE", volume qu vient de paraitre à Toulouse et dont on trouvera plus loin l'analyse biblographique, quelques notes qui présentent beaucoup dintérêt pour les lecteus de La Houlle Blanche. Elies complètent les confetences et comptes rendus que nous avons déjà publiés l'année dernè̀e au moment de ce Congrès (1).

Votci d'abord textuellemont la communication de M. MARCHAND, dintefer de lobserzatoure du Puc du Midt.

\section{LENERGIE MÉCANIQUE TOTALE SUR LE VERSANT FRANÇAIS DES PYRÉNÉES}

"On peut obtenir assez facilement une évaluation approchée de cette énergie par une méthode dont je me borne à indiquer ici le principe.

"En un point quelconque du versant français des Pyrénées, il tombe annuellement une quantité d'eau (pluie ou neige) qui dépend surtout de l'altitude de ce point. Cette eau est en parlie évaporée, mais une partie importante ruisselle sur les pentes du versant, descend du point où elle est tombée jusqu'à un niveau inférieur (celui des plaines sous-pyrénéennes ou celui de la mer), en développant un travail qu'il est facile d'évaluer quand on connaît le poids de cette eau et la différence de niveau entre le point où elle a été recueillie et le point où eile arrive finalement. On peut établir des relations mathématiques approchées : I $^{\circ}$ entre l'altitude d'un point et la hauteur annuelle de pluie ou neige recueillie sur ce point (d'après les observations pluviométriques) ; $2^{\circ}$ entre l'altitude d'un point et le travail mécanique développé par la descente des eaux pluviales reçues en ce point jusqu'à un niveau inférieur domé.

"Ces relations étant établies pour un point, on peut ensuite chercher le travail total développé par la descente des caux sur les pentes d'un versant dont les dimensions et les différences de niveau sont données.

"Dans cette évaluation, dont le but n'est que d'arriver à des résultats approchés, on néglige les détails des reliefs pour ne considérer que des plans inclinés partant des crêtes de la haute chaîne et aboutissant aux niveaux de plaine ; il y a alors compensation (dans les limites de l'approximation à obtenir) entre les divers détails de reliefs négligés.

"En faisant cette "intégration " approximative pour les diverses régions du versant français des Pyrénées, on arrive rapidement à se faire une idée de l'ordre de grandeur de l'énergie totale des eaux qui ruissellent sur l'ensemble du versant.

(1) Voir La Honille Blanche. Conférence de M. Ader sur: "La Houille blanche dans les Pyrénées ", jun I9o3, page 137 . "A propos de la dégradation du Puy-de-Dóme b, B. Brunies, meme numéro, page 167 . "La lutte pour et contre l'eau n, I..-A. Farre, même numéro, page 177 . "La question du reboisement des Pyrénées au Congrès du Sud-Ouest-Navigable ", S. GuÉNor, juillet rgo3, page 190. "Observations sur le régime des gaves de la région tiêres "P. Bugfaurs, même numero, page 203 . "Nos lois forestieres $\$$ P. Buffault, septembre 1903 , page 275 . Etc.
" Je répète qu'll ne s'agit pas, par cette méthode, d'obtenir un résultat précis, dont l'intérêt serait pour le moment asser. secondaire; il s'agit seulement d'arriver à quelques chiffres frappants, montrant l'énorme valeur de l'énergie développée par le ruissellement des eaux dans les Pyrénées, énergie qui est actucllement presque entièrement perdue.

"On doit naturellement déduire du travail ainsi calculé celui qui correspond à la portion de l'eau qui s'évapore. On devrait aussi (pour arriver à évaluer le travail pratiquement utilisable) en déduire l'énergie des ruissellements supérieurs.

"Il est relativement facile de faire la première déduction; il est beaucoup plus difficile de faire la seconde.

"Voici les résultats auxquels on arrive : déduction faite de l'évaporation, l'énergie totale áes eaux qui descendent sur le versant français des Pyrénées est, en chiffres ronds, de 8 mallions de chevaux-vapeur.

"On s'en fera une idée plus concrète en remarquant qu'elle correspond au travail de 25000 locomotives (supposées de 300 chevaux chacune) circulant nuit et jour.

"Quant à l'énergie pratiquement utilisable, il est fort difficile de l'évaluer; le mot " pratiquement " n'a, en effet, qu'un sens tout relatif, car telle chute d'eau considérée actuellement comme difficile à explonter sera peut-être utilisée dans la suite des temps, lorsque toutes les chutes placées dans les meilleures conditions auront été successivement mises au service de l'industrie.

"Le fait à retenir pour le moment est que la " houille blanche ", dans les Pyrénées françaises, développe assez. d'énergie pour mettre en mouvement un nombre colossal d'usines $n(\mathrm{r})$.

(1) Il est instructif de rapprocher de ce calcul les renseignements fournis par M. Aner, ingénieur des Ponts-ct-Chaussées à Narbonne, daus sa conférence au même Congrès et que nous avons publiée in extenso dans le no de juin 1903 .

Au sujet de ce que l'on connuit de ces forces, il dit : "ll n'a jamais été fait d'évaluation générale des forces motrices disponibles dans les Pyrénées. Quant aux forces déjà aménagées, elles ont fait lobjet d'un travail statistique très important dressé par le Service de l'Hydrauhque agricole et dont les résultats généraux ont été analysés par M. Turquan.

"De ce travail il résulte que, dans les six départements frontières (Aliege, Aude, Haute-Garonne, Basses-Pyrénées, Hautes-Pyrénées et Pyrenées-Orientales), les usines hydrauliques existant on 1800 sur lcs rivières non navigables ni flottables, représentaicnt une puis. sance brute totale de 93401 chevaux, répartie en 5728 usines dont la puissance moyenne n'était ainsi que de 17,7 chevaux-vapeur; les machines à vapeur installees dans la même région ne représentaient qu'une puissance totale' de 16229 chevaux.....

"Nouvelle appréciation sommaire. Ce n'est que par un travail tres important et de longue haleine que l'on pourrait arriver a etre fixé exactement sur limportance des chutes d'eau pyrénéennes susceptibles d'être pratiquement aménagées, et il scrait tout à fait téméraise de vouloir des maintenant en tenter l'évaluation. 11 m'a paru cependant intéressant de tâcher de réunir quelques éléments dappréciation de la situation d'ensemble de nos cours d'eau. Tout d'abord, jai fait une estimation tres sommaire de la puissance potenticlle disponible, en temps d'étiage, dans chacun des bassins pyrénéens, sans

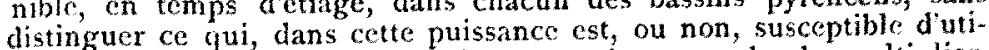
lisation industriclle; je me suis contente pour cela de multiplier, pour chaque cours d'eau, le débil d'étiage par la difference d'alti- 


\section{DE L'EMPLOI DES FORCES HYDRAULIQUES DU LOT PAR L'ÉNERGIE ÉLECTRIQUE}

Résumé de la communication de M. L. Autesserre, vice-président du Comité du a Lot navigable $D$

L'auteur veut amener les pouvoirs publics à donner satisfaction aux intérêts économiques desquels dépend la prospérité des départements traversés par le Lot et qui ont pour facteur indispensable la reconstitution de la navigabilité de rette rivière

Les documents statistiques de la consommation de houille sur la place de Bordeaux accusent annuellement un million de tonnes sur lesquels un dixième à peine est de provenance française; tout le reste, soit 900000 tonnes environ est du charbon anglais. Ce fâcheux état de choses subsiste depuis de longues années, bien que la Compagne d'Orléans, en vue de concurrencer la production étrangère, at abaissé le tarif plein de Decazeville à Bordeaux, qui est de 13 francs par tonne, à $\sigma \mathrm{fr}$. 50 , solt à son extrême limite; au-dessous de ce prix, la Compagnie serait en perte.

M. Autesserre a la preuve matérielle que, si le prix pouvait ne pas dépasser 5 francs par tonne, la houille française entrerait au moins pour moitué dans la consommation de la place de Bordeaux. Or, d'un projet étudié par les ingénieurs les plus compétents, il résulterait qu'on peut compter abaisser le prix de transport de Bouquiès à Bordeaux au-dessous de 4 francs par tonne, tout en réservant un bénéfice de I fr. par tonne à l'entreprise de traction électrique.

Ce résultat si désirable, non seulement dans l'intérêt des localités industrielles des houllères de l'Aveyron, aux portes du département du Lot, mais encore et surtout dans celui cles populations de la vallée de la ruvière du Lot, serait certainement obtenu en utilisant la voie navigable; on ne peut, en effet songer à utıliser la vore ferrée, attendu qu'au-dessous de $6 \mathrm{fr}$. 50 par tonne la Compagnie perdrait la différence. C'est une erreur de croire à une hostilité de la part de la Compagnie d'Orléans; la rivière n'est pas l'ennemie du chemin de fer, elle doit être au contraire son auxiliaure.

Les deux voies de transport ne répondent pas absolument aux mêmes besoins : le chemin de fer est destiné au transport des marchandises peu encombrantes, d'un poids relativement faible et qui demandent une certaine rapidité soit par leur nature, soit par leur destination. La rivière, au contraire, sera réservée aux marchandises lourdes, encombrantes, d'une valeur peu importante, et qui n'exigent pas un transport rapide. Tel serait le cas pour les charbons, les bois, les matériaux de construction et les produits de l'agricuiture de la réginn, qui se transportent en grande masse et ne risquent pas de se détériorer pendant le trajet. Le fret par chemin de fer étant beaucoup plus élevé que par bateau, obliger ces produits à emprunter la voie ferrée est presque toujours un obstacle, sinon un empêchement absolu, à leur transport : la batellerie de la rivière en souffrira et le chemin de fer n'y gagnera rien. Si donc la navigabilité et la batellerie du Lot étaient rationnellement organisées, cette dernière pourrait prendre

tude entre son extrémité aval et le niveau moyen du bassin versant (limité au-dessus de la cote 200 mètres). Mon calcul n'a, d'ailleurs, porté que sur les rivières pyrénéenpes proprement dites et j'al laissé en delrors celles qui descendent du Platcau central. Je suis ainsi arrivé ì un tolal de I 200 oxo chevaux-rapeur, qui correspondent sersiblement à une puissance de 2000 o00 chcvaux en caux moyennes.....

"Jai fait ensuite unc enquete très minutieuse dans les divers centres pyrénéens pour évaluer, d'une part, l'importance des chutes supérieures a conchcraux deja amenagees et, dautre part, celles qui ont déjà été envisagées comme susceptibles d'aménagement pratique imméduat et pour lesquelles les études sur le terrain ont été faites.

"Jo suis arrivé, pour le's premières, à un total cle 31837 chcvaux, répartis en 90 usincs, et pour les secondes 133500 chevaux, répartis en 33 chutes..." dans le bassin houiller de I'Aveyron et dans les centres de tout son parcours les charbons, les marchandises encombrantes et les tranpotterait à Bordeaux où elles n'arrivent pas aujourd'hui et, au retour, ramener des engrais, des boss du Nord et bien d'autres produits qui demeurent perdus ou font supporter à l'agriculture de la région des frais de transport écrasants.

L'auteur se demande donc $s$, indépendamment de l'insuf. fisance reconnue de l'état de navigabilité du Lot, la situation, au point de vue matériel, de sa batellene permettrait développement efficace à la navigation qu'il réclame. Il tie suffit pas, dit-il, de créer des canaux, d'amélio,er des cours d'eau, 1 faut encore que la batellerie sache bien tirer parti des ressources nouvelles mises à sa disposition.

C'est une industric encore dans l'enfance qui doit transfor. mer les modes primitifs de traction, dont elle a fait jusqua ces derniers temps un usage à peu près exclusıf. Le halage à la corde, à traction de cheval ou de mulet, parfois même avec traction d'hommes, sont des modes trop lents, couteux, vorr même inhumains. Une réforme absolument nécessaire s'impose : c'est la substitution de la traction électrique à ces no. des de traction archaiques. Avec la traction animale la vitesse moyenne kilométrique, compris le passage des écluses est de I 200 mètres à l'heure; elle n'est que de 850 mètres par le halage à bras d'homme. M. Berget, ingénieur en chef de la Navigation du Lot, estime qu'avec son système de traction électrique on atteindra une vitesse commerciale de 3,500 mètres minimum à l'heure.

L'électricité, qui a déjà réalisé tant do merveilles, dôt réussir là où les moteurs animés - l'homme, les bêtes de trait et de somme -- ont échoué; quoique cette force solt encore incomplètement disciplınée, partout les inventions se succèdent plus surprenantes les unes que les autres. Des forces naturelles délaissées pour la houille noire vont être de plus en plus utilisées. "En créant des usınes électriques sur notre " ruvière, il nous sera facıle, après avorr distrıbué aux accu" mulateurs de notre batellerie l'énergie nécessaire, d'appor. " ter ici la lumière, là la force motrice soit à des lignes de tramways, soit à de grandes usines, soit encore à une infinité de petites industries, de petits ateliers... Quel progrés le jour où nous serons parvenus à utiliser à grande distance les chutes d'eau, forces naturelles de notre rivière inutil " sées !... Ce sera toute une révolution économaque et socale "dans maintes branches du traval industriel; l'atelier domestique sera reconstitué et avec luı la vie de famile, "principal élément de bonheur et de prospérité. "

L'utilisation de la rivière du Lot qui, par sa situation, son étendue, ses conditions de pente et de débit, constitue un moyen de transport bénéficiant d'avantages naturels des plus remarquables, était tout indiquée. Aussi n'est-1l pas surprenant que les ingénieurs se soient attachés à retirer tout le parti possible de cette situation, en complétant l'œuvre de la nature par un certain nombre d'améliorations indispensables à sa navigabilité. On a d'abord effectué des travaux de régularisation très importants; on s'est efforcé ensute d'unfier la profondeur du lit de la rivière sur tout son parcours, de manı̀̀re à permettre la marche régulière des bateaux.

Malgré ses multiples avantages, ses nombreux barrages et ses superbes écluses, la navigation du Lot périclite depuis I 865 , c'est-à-dire depuis l'époque de l'ouverture des chemins de fer. C'est ainsi qu'on voit le tonnage transporté sur le Lot tomber de I91 676 tonnes en I 857 à 33994 tonnes en 1894 La décroissance de la navigation parait donc bien correspon. drè à l'ouverture des voies ferrées. "Doit-on, dit M. Autes" serre, abandonner la dépense de 21 millions et demi qu "ont été absorbés par les travaux d'amélioration et de ca"nalisation de notre rivière et laisser continuer les frais d'entretien s'élevant à la somme annuelle de 220000 francs d'entretien s'élevant à la somme annuelle de 220000 fra de
environ?" Non, répondit-il. On a prétendu que le rôle de 
la navigation du Lot était fini, à cause de la supériorité des chemins de fer. C'était, ajoute-t-il, une illusion excusable lors de l'enthousiasme excité par les premiers succès des vores ferrées, mais aujourd'hui on s'accorde unanımement à reconnaitre que les voies navigables et les chemins de fer sont destinés non à se supplanter, mais à se compléter.

De Bouquiès (Aveyron) à Aiguillon (Lot-et-Garonne), le Lot offre une voie navigable de 256 kilomètres; sur ce parcours, il existe 73 écluses. A Bouquiès, la rivière coule à la cote d'altitude 170 ; à Cahors, à la cote 122 ; à Fumel, 65 ; ̀̀ Aiguillon, 22; soit une dénivellatıon totale de 150 mètres.

Le débit du Lot à l'étiage est de 10 mètres cubes environ; la force motrice brute tolale que représente l'ensemble des hutes d'eau créées sur le parcours indiqué est en nombre rond de 20000 chevaux; un quart au plus de cette puissance respeclable, soit 5000 chevaux seulement, est concédé à l'industrie. La puissance moyenne créée par chaque barrage est de 275 chevaux environ à l'étıage.

Par son extrémité amont, le Lot canalisé traverse le bassin houller de l'Aveyron dans toute sa longueur : la production annuelle atteint plus d'un million de tonnes et peut être dépassée dans une large mesure (il y a dans le bassin wo millions de tonnes de charbon reconnues); par son extrémité aval, il débouche dans la Garonne, à proximité de Bordeaux. Cette ville consomme environ un million de tonnes de houille.

Voilà, certes, une situation heureuse et pleine de promesses. Que les charbons étrangers solent chassés de Bordeaux ce qui sera possible avec la nouvelle batellerie, car l'envahissement continu des marchés de notre pays par les houilles anglases provient uniquement des prix de transport trop élevés auxquels sont assujettis les charbons français - ce sera alors 300 ooo tonnes que le bassin houiller de l'Aveyron produra en plus, et c'est 2 millions de salaires que gagneront en plus les mineurs de la région.

Dans un remarquable projet, $M$. Berget se borne à faire ressortur les services que doit rendre la batellerie nouvelle et les avantages qui en seront la conséquence. Mais, comme le fait remarquer M.Autesserre, la Chambre de Commerce représentant les intérêts industriels et commerciaux du département doit assumer la mission d'agrandir le rayon d'action de ce projet. Il y a lieu, dit-il, d'y ajouter l'emploi de cette puissance de 15000 chevaux non utilisés, puissance qui sera si bien employée à l'éclairage des villes et des centres d'habitation situés sur le parcours de la rivière et qui en outre, distribuée partout pour l'utilisation industrielle, donnera à la contrée une anmation inconnue jusqu'à ce jour.

Aux nombreuses ressources que fera renaitre sur son parcours la batellene électrique viendra donc s'ajouter le produit des usines qui $y$ seront construites. M. Berget en indique six dans son projet, mais 11 sera peut-être bon d'en établir davantage, car la Compagnie concessionnaire trouvera là quantité d'éléments de rémunératıon, ainsi que le prouve le produit de celle déjà construite sur le Lot à l'écluse de Capdenac.

Cette usine distribue : $I^{\circ}$ I.a lumière à Capdenac, ville et gare, aux villes de Penchot, Viviez, Aubin, le Gua et Cran$\mathrm{sac}$, et bientôt à Decazeville et même à Frgeac; 2 "l'énergie électrique à des industriels de Capdenac, aux moteurs amenant l'eau à la gare des voyageurs, aux ateliers et au dépôt, aux plaques tournantes, gare et machines, aux laminö̈rs de l'usine à zinc de Pe nchot, et à l'usine à zinc de la VienlleMontagne, à Viviez. Cette première usine électrique qui utilise à peine 200 chevaux de force réalise déjà, dit-on, $52000 \mathrm{fr}$. de recettes dès la troisième année. Ceci laisse à prévoir le bénéfice qu'on pourra retirer de l'utilisation de toute l'énergie de la rivière.

Des études très sérieuses démontrent que le J.ot, dans peu de temps, reviendra facilement à la vie et qu'en rétablissant sa navigabilité, la batellerie nouvelle, transformée par la traction électrique, reprendra un essor inconnu sur ses rives. La création d'usines électriques sur son parcours ne manquera pas en outre de fare naître partout, par l'utulisntion industrielle, des éléments d'activité qui développeront : popu lation, rıchesse et fertılité là où règnent maintenant la solitude et la plus profonde misère'...

\section{TACHYMÉTRE ENREGISTREUR}

\section{du Commandant AUdebrand}

Les compteurs de tours ordinaires sont parfois chers, souvent inexacts et, tout en fatiguant l'attention de l'opé. rateur, ne laissent pas de traces de leurs lectures. Les appareils enregistreurs combinés jusqu'ici sont en général délicats, peu faciles à transporter ou à improviser, et d'un prix élevé. M. le commandant Audrbrand, bien connu de nos lecteurs, s'est préoccupé de ces difficultés d'ordre pratique et s'est proposé de construire un appareil peu coûteux, facile à improviser en tout lieu, et capable de donner le nombre de tours d'un arbre horizontal, avec une approximation au moins égale à celle qu'exige la pratique industrielle.

A cet effet il cale sur l'arbre $C$, dont il veut connaître les tours, un disque vertical en bois blanc recouvert d'une

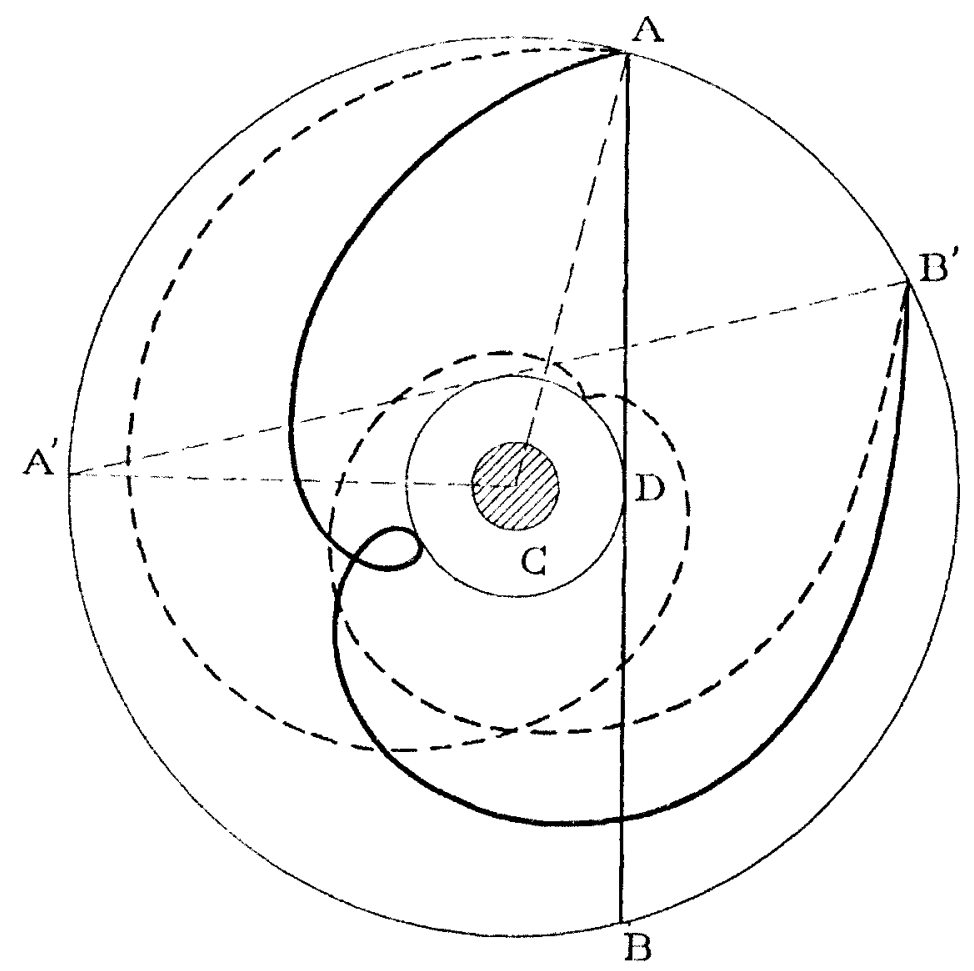

feuille de papier. Devant ce disque, et selon une verticale $A B$ située à une distance $C D$ du centre $C$, sensiblement égale au $1 / 4$ du rayon du disque, il fait tomber d'une hauteur $h$, choisie dans chaque cas au-dessus du point $\mathbf{A}$ du disque, un poids porteur d'un pinceau imbibé d'encre. Au moment où le pinceau passe devant le disque son mouvement vertical se combine avec la rotation de ce disque. Il s'inscrit des lors sur la feuille qui couvre celui-ci une courbe dont le commencement correspond à l'instant où le pinceau était au niveau $\mathrm{A}$ et dont la fin marque celui où il était au niveau $B$. Or rien n'est plus aisé que de mesurer l'arc A A'B B' A A' décrit par le disque pendant 